

\title{
Focusing light through living tissue
}

\author{
Vellekoop, I M ; Aegerter, C M
}

\begin{abstract}
Tissues such as skin, fat or cuticle are non-transparent because inhomogeneities in the tissue scatter light. We demonstrate experimentally that light can be focused through turbid layers of living tissue, in spite of scattering. Our method is based on the fact that coherent light forms an interference pattern, even after hundreds of scattering events. By spatially shaping the wavefront of the incident laser beam, this interference pattern was modified to make the scattered light converge to a focus. In contrast to earlier experiments, where light was focused through solid objects, we focused light through living pupae of Drosophila melanogaster. We discuss a dynamic wavefront shaping algorithm that follows changes due to microscopic movements of scattering particles in real time. We relate the performance of the algorithm to the measured timescale of the changes in the speckle pattern and analyze our experiment in the light of Laser Doppler flowmetry. Applications in particle tracking, imaging, and optical manipulation are discussed.
\end{abstract}

DOI: https://doi.org/10.1117/12.841159

Posted at the Zurich Open Repository and Archive, University of Zurich

ZORA URL: https://doi.org/10.5167/uzh-40291

Conference or Workshop Item

Originally published at:

Vellekoop, I M; Aegerter, C M (2010). Focusing light through living tissue. In: Optical coherence tomography and coherence domain optical methods in biomedicine XIV, San Francisco, CA, USA, 25 January 2010 - 27 January 2010. Spie - The International Society For Optical Engineering, 755430 (10 pp.).

DOI: https://doi.org/10.1117/12.841159 


\title{
Focusing light through living tissue
}

\author{
I. M. Vellekoop and C. M. Aegerter \\ Physics Institute, University of Zurich, \\ Winterthurerstrasse 190, CH-8057 Zurich, Switzerland
}

\begin{abstract}
Tissues such as skin, fat or cuticle are non-transparent because inhomogeneities in the tissue scatter light. We demonstrate experimentally that light can be focused through turbid layers of living tissue, in spite of scattering. Our method is based on the fact that coherent light forms an interference pattern, even after hundreds of scattering events. By spatially shaping the wavefront of the incident laser beam, this interference pattern was modified to make the scattered light converge to a focus. In contrast to earlier experiments, where light was focused through solid objects, we focused light through living pupae of Drosophila melanogaster. We discuss a dynamic wavefront shaping algorithm that follows changes due to microscopic movements of scattering particles in real time. We relate the performance of the algorithm to the measured timescale of the changes in the speckle pattern and analyze our experiment in the light of Laser Doppler flowmetry. Applications in particle tracking, imaging, and optical manipulation are discussed.
\end{abstract}

Keywords: Wavefront shaping, interference, turbid media, focusing

\section{INTRODUCTION}

A wide range of methods is available for biomedical imaging. Choosing the best imaging method for a given problem usually involves a tradeoff between imaging depth, resolution, speed, specificity, and cost. Optical methods are superior on almost all of these points. With light it is possible to achieve sub-micrometer resolution, study high speed processes such as hemoglobin concentration fluctuations ${ }^{1-3}$ and calcium uptake ${ }^{4}$ in the brain, have spectrometric sensitivity and to use intrinsic or extrinsic markers such as fluorescent molecules. ${ }^{5}$ Moreover, the cost of optical methods is generally low compared to CT, PET or MRI. Compared to these methods, light has the extra benefit of allowing active manipulation of the subject by means of optical microsurgery, ${ }^{6}$ by applying mechanical forces with optical tweezers, ${ }^{7}$ or by photochemical activation of drugs, ${ }^{8}$ genes, ${ }^{9}$ or brain activity. ${ }^{10}$

The only point where optics looses the competition with other imaging methods is the penetration depth in turbid tissue. Most optical methods cannot penetrate deeper than a couple of hundred microns into living tissue. The reason for this limitation is that inhomogeneities in the tissue scatter light in random directions, preventing the formation of a focus or a sharp image. At the moment, this restriction is prohibitive for most in-vivo applications. Therefore, a method for reducing the effects of scattering would be highly useful.

In this article we demonstrate that it is possible to counteract strong scattering in living tissue. We present the first application of wavefront shaping to focus light through a living animal. Wavefront shaping is a recently developed technique for guiding light through scattering materials by spatially shaping the wavefront of the incident laser beam. ${ }^{11}$ Using wavefront shaping, the effects of scattering can be 'canceled' and the light can be made to focus right through the tissue. Ultimately, this method has the potential of dramatically increasing the penetration depth of optical imaging and manipulation methods in turbid tissue.

To place our results in context, we first discuss existing state-of-the-art methods for imaging in turbid media in Sec. 1.1. These methods are passive in the sense that no attempt is made to guide the light through the scattering medium. In contrast, wavefront shaping uses interference of scattered light to actively guide light through scattering media. In this aspect, wavefront shaping is similar to phase conjugation and time-reversal, so these methods can be summarized as 'interferometric focusing'. In Sec. 1.2 we summarize the most recent results in interferometric focusing. In Sec. 2 we analyze how the movement of scattering particles in living tissue affects wavefront shaping and discuss parallels with laser Doppler flowmetry (LDF). Sec. 3 describes our experimental apparatus and samples. Our experimental results are presented in Sec. 4. Possible applications of our method are discussed in Sec. 5.

Further author information: (Send correspondence to I.M.V.) I.M.V.: E-mail: ivo.vellekoop@physik.uzh.ch 


\subsection{State of the art}

There have been tremendous efforts in developing methods for imaging in scattering tissue. We can divide existing optical methods into two categories. Methods in the first category use light that has not been scattered (the so called ballistic light) to form an image. The challenge is to separate the ballistic light from the diffuse background. The penetration depth of all such methods is fundamentally limited due to the fact that the ballistic component of the light decreases exponentially with depth. The second category, that of diffuse tomographic methods that use the diffuse light to form an image, will be discussed briefly in Sec. 1.1.5

\subsubsection{Confocal microscopy}

In confocal microscopy, light from a source is focused to a single point in the specimen. A detector selectively measures reflected light or emitted fluorescence coming from that point. Light coming from other points (scattered light or the out-of focus contribution) is blocked by a pinhole. By scanning the focus through the specimen, a 3-dimensional image is formed.

Although the first confocal microscope worked in transillumination, ${ }^{12}$ the most common systems today are epifluorescence microscopes. The confocal epifluorescence microscope is widely used for studying developmental processes and processes taking place in single cells. The main benefits of this type of microscopes are depth specificity that allows full 3-dimensional imaging, and the ability to measure fluorescence. Fluorescence is used, for instance, for in-vivo imaging of gene activation through genetic constructs involving Green Fluorescent Protein (GFP). ${ }^{13}$

The major disadvantage of confocal microscopy is the limited imaging depth. Since the microscope only uses the ballistic fraction of the light, the signal strength decreases exponentially with depth. Another disadvantage is that the collection of emitted photons is very inefficient: most emitted photons correspond to an out-of-focus contribution of the light and are blocked by the pinhole. This lack of efficiency is a problem because phototoxicity and bleaching limit the exposure time and intensity of the pump laser. In small, transparent specimens, a significant increase in the detection efficiency can be reached through selective plane illumination microscopy (SPIM). ${ }^{14,15}$

\subsubsection{Two-photon excitation microscopy}

Two-photon excitation microscopy ${ }^{16}$ tackles two important problems in confocal fluorescent microcopy: limited penetration depth and phototoxicity. The two-photon microscope uses an excitation source with a long wavelength. Fluophores are excited by simultaneous absorption of two source photons. Since the excitation probability is proportional to the squared intensity of the source light, excitation only takes place right in the focus of the excitation light. This effect makes that bleaching and phototoxicity are greatly reduced. Moreover, longer wavelengths are generally scattered less, allowing imaging at increased depths. Still, the signal decreases exponentially with depth and the excitation intensity cannot be increased indefinitely because of thermal damage.

\subsubsection{Optical coherence tomography}

Optical coherence tomography (OCT) is a marker-free imaging method that is able to image up to about ten optical mean free paths deep. ${ }^{17}$ OCT uses coherence gating to isolate 'early photons' corresponding to the minimally scattered light. Although OCT is exceptionally good at separating the signal from the diffuse background, this method still relies on the presence of a ballistic component of the light: when all light is scattered, even OCT fails.

\subsubsection{Adaptive optics}

Adaptive optics (AO) is not an imaging method of its own, rather it is a technique to improve the resolution of an imaging method by correcting aberrations in the imaging optics and/or gradual refractive index variations in the specimen. ${ }^{18}$ Adaptive optics is not designed for correcting scattering on sub-wavelength particles or for dealing with multiply scattered light. Therefore, imaging is still based on the ballistic component of the light and AO can not be used to prevent the diffusion of light. 


\subsubsection{Diffuse tomography}

Imaging methods that use the diffuse light, rather than the ballistic light, to form an image are summarized as diffuse tomography (DT). In DT intricate algorithms are used to invert the diffusion equation and find out where the diffuse light most likely originated. ${ }^{19}$ Common examples of DT include fluorescence tomography, capable to image at few-millimeter resolution through about ten centimeter of human tissue ${ }^{20}$ or at sub-millimeter resolution through small animals, ${ }^{21}$ as well as diffuse light spectroscopy used for imaging blood flow in the brain. ${ }^{1-3}$ Because DT uses diffuse light for imaging, it can image much deeper into turbid tissue than microscopic methods can. Unfortunately, the increased depth comes at the cost of a decreased resolution. State of the art methods have a resolution ranging from hundreds of microns to millimeters. ${ }^{5}$

\subsection{Interferometric focusing}

Ideally, one would want to combine the resolution of ballistic imaging methods with the imaging depth of diffuse tomography. A promising step towards achieving this 'holy grail' of imaging in turbid media is taken by the development of methods that we summarize as 'interferometric focusing'. With interferometric focusing light can be made to focus right through a scattering layer. In contrast to focusing with a lens, interferometric focusing does not rely on light propagating along a well defined path. Rather, it uses interference of scattered light to form a focus. Even when all the light has scattered multiple times a focus can be formed.

When a laser beam is incident on a scattering medium, the scattered light forms a random interference pattern known as laser speckle. A different beam, incident at a different angle or at a different position, gives rise to a completely different speckle pattern. When multiple coherent beams are incident simultaneously, the resulting speckle fields interfere. By modifying the relative phase of the incident beams, we can make the fields interfere constructively at any chosen point, effectively focusing the scattered light to that point. The more independent beams are used, the higher the intensity in the focus will be. ${ }^{11}$ In practice, instead of using independent beams, a single beam with a spatially shaped wavefront is used. The intensity in the focus then depends on the number of controlled optical degrees of freedom in the incident beam.

The crux of this method is constructing the incident wavefront that makes the light focus to the desired point. We now discuss several known methods for constructing a shaped wave that focuses through a strongly scattering turbid medium.

The oldest known method for interferometric focusing is optical phase conjugation (OPC). ${ }^{22}$ In OPC, light is sent through the scattering layer twice. First a coherent light source is placed at the position where we want the light to focus. The light that is transmitted through the scattering layer is collected and reflected off a phase conjugating mirror (PCM). The PCM conjugates the phase of the optical wave and sends it back in the direction where it came from. Thanks to the reciprocity of light propagation, the conjugated wave refocuses after traveling back through the scattering medium. Recently, the feasibility of using PCM in biological tissue has been demonstrated. ${ }^{23}$

The main limitation of OPC is that it requires having a coherent light source at the point where the light is to refocus, $\mathrm{i}$. e. direct optical access to the focal point is required. Since we are interested in guiding light to points where we don't have direct optical access to (in or behind a scattering layer), this limitation of OPC makes it unsuitable for most biomedical applications. Fortunately, there are alternative methods for constructing the shaped wave that leads to interferometric focusing. Noteworthy is time-reversal, which can be thought of as phase conjugation in the frequency domain. Timereversal is highly effective for micro waves, ${ }^{24}$ radio waves ${ }^{25}$ and ultrasound. ${ }^{26}$ Time-reversal of optical waves, however, is technically very challenging. Moreover, this method, too, requires having a light source at the point where the light is to be refocused.

At the moment, the only method that does not require having a light source in the desired focal point is feedback-based wavefront shaping ${ }^{11,27,28}$ (See Fig. 1). Wavefront shaping uses feedback from a detector or a fluorescent probe particle behind or inside of the scattering tissue. Especially the ability to focus light on fluorescent markers embedded deep inside turbid materials makes this method promising for biomedical applications.

Ideally, with wavefront shaping one would focus light on an injected fluorescent probe particle, or focus on individual cells that are tagged with fluorescent antibodies or genetically modified to produce fluorescent proteins such as GFP. Once the focus is formed, the light might be used to:

- Trap the particle or the cell by using the focus as optical tweezers.

- Thermally activate genes or destroy the cell by increasing the laser power after optimization. 
a

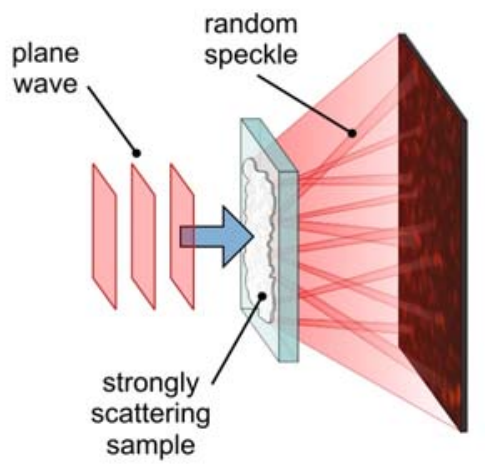

b



Figure 1. Principle of interferometric focusing using wavefront shaping (reproduced from Ref. 11). (a) Normal situation. An incident light beam is scattered by inhomogeneities in the turbid medium. The scattered light forms a disordered interference pattern. (b) After running the optimization algorithm, the incident wave exactly matches the scattering 'fingerprint' of the medium. The scattered light that makes it through the medium interferes constructively at the chosen point, effectively creating a focus.

- Optically activate genes or photosensitive chemicals in the vicinity of the cell.

- Track a slowly moving cell as it moves deeper into the tissue.

- Improve the resolution of diffuse tomography: since the diffuse light is guided towards the focus it spreads out less, so the effect of diffusion is reduced. This effect might be strong enough to improve the resolution of DT and fluorescence tomography.

The main limitation of wavefront shaping and all other interferometric focusing methods is the requirement for the scattering medium to be static during the course of the measurement. At the moment, the timescale of wavefront shaping is seconds to minutes. In our experiment, we demonstrate that this process is fast enough to focus light through a complete living animal (the pupa of a Drosophila melanogaster). Our results show that, already now, wavefront shaping can be used in living tissue. In Sec. 5 we discuss how future wavefront shaping setups can benefit from technological advances that allow a speedup of over a million times.

\section{WAVEFRONT SHAPING FOR A NON-STATIONARY MEDIUM}

The principle of wavefront shaping is illustrated in Fig. 1. We consider a situation where a layer of turbid material completely scatters all incident light (Fig. 1a). Both the reflected light and the transmitted light form random interference patterns, known as laser speckle. By placing a spatial light modulator in the incident beam, we can locally retard or advance the beam. When the wavefront of the incident light is modified, the interference pattern of the transmitted light changes. By monitoring the intensity in the desired focal point, a learning feedback algorithm (see Sec. 2.1) is able to find the optimum configuration at which the transmitted light interferes constructively in the desired focus (Fig. 1b).

Optimizing the incident wavefront can increase the intensity in the focus significantly. The intensity of the focus is quantified as the enhancement $\eta$, which is defined as the ratio of the intensity in the optimized target to that of the diffuse background. In experiments with solid samples, enhancements of over 1000 were reached. ${ }^{11}$ However, the optimum configuration of the light modulator strongly depends on the position of all scattering particles in the medium. Since in living tissue the particles are not perfectly stationary, the optimum configuration is time dependent. Therefore, to keep the light focused, it is required to dynamically track the changes in the speckle pattern. The enhancement will depend on how well these changes can be tracked and it will be significantly lower than results for solid materials. To analyze the effect of moving particles, we first explain the algorithm that was used and then discuss how dynamic wavefront shaping relates to Laser Doppler flowmetry, a well known method for measuring movement in turbid media. 


\subsection{Algorithm}

In our experiments, we used the partitioning algorithm as discussed in Ref. 29. This algorithm randomly divide the 2500 segments of the phase modulator over partitions $A$ and $B$, each containing approximately half of the segments. The phase of partition $B$ is varied with respect to partition $A$ and the phase shift that corresponds to the highest value of the feedback signal is kept. After a single such iteration, the algorithm continues indefinitely, choosing different partitions for every iteration.

Because the wave is optimized continuously, the partitioning algorithm dynamically follows changes in the speckle pattern. How well the changes can be followed is quantified by the ratio of the persistence time of the speckle $T_{p}$ (see Section 2.2) to the time needed for a single iteration of the algorithm $T_{i}$. The theoretically achievable enhancement for this algorithm equals ${ }^{29}$

$$
\eta=0.5 T_{p} / T_{i}
$$

when the number of modulator segments $N$ is large enough $\left(N \gg T_{p} / T_{i}\right)$. The partitioning algorithm gives close to optimal performance, regardless of the rate of change in the sample or any other system parameter. ${ }^{29}$ Moreover, since the algorithm always adjust the phase of a maximum number of segments simultaneously, it rapidly forms an initial focus and it recovers from disturbances quickly. The enhancement only depends on the persistence time and it will automatically increase at moments when scatterers move less.

The drawback of adjusting so many segments at once is that a single measurement error can destroy the entire focus: when the measured phase in a single iteration is $\pi$ off, the intensity in the focus will be canceled in a single iteration and many iterations are required to build it up again. To prevent these types of 'catastrophes', the algorithm remembers the last known successful configuration. When the intensity has decreased over $5 \%$ after a single iteration, the algorithm reverts to the stored configuration.

\subsection{Laser Doppler flowmetry}

When scatterers inside a turbid medium move, the speckle pattern of reflected or transmitted monochromatic light changes in time. There are several well known methods for characterizing the rate of change in the speckle pattern, thereby giving semi-quantitative information about the amount of movement in the specimen.

One of these methods is known as diffusing wave spectroscopy (DWS). ${ }^{30,31}$ In DWS, the intensity at a single point in the speckle pattern is recorded as a function of time. Subsequently, the autocorrelation function of this signal is computed, whose width gives a characteristic time scale for the movement.

A different interpretation of the same physical phenomenon is obtained when one considers that the light that is scattered by a moving particle is Doppler shifted: ${ }^{32}$ the scattered light will have a slightly different frequency and wavelength than the incident light. This interpretation forms the foundation for Laser Doppler flowmetry (LDF). With LDF, the dynamically scattered light interferes with a stationary reference beam. The Doppler shift, which is a measure for the amount of movement in the sample, is deduced from the beating frequency of the two waves.

Whether we look at the signal in the time domain as in DWS or in the frequency domain as in LDF, the underlying physics is the same. ${ }^{32}$ The only real difference between the methods is that LDF uses a reference beam and, therefore, measures changes in the speckle field, whereas DWS measures changes in the speckle intensity. In a strongly scattering medium, the correlation function of the transmitted intensity is simply the square of the correlation function of the field, ${ }^{31}$ so both methods will yield comparable timescales.

In our experiment, we measured the rate of change in the speckle pattern by recording the time varying speckle pattern with a CCD camera. This measurement can be thought of as performing a DWS measurement for each pixel on the camera in parallel. ${ }^{33}$ For each frame, we calculated the cross correlation between that frame and the initial frame using

$$
C(\tau)=\frac{\sum_{i}\left[I_{i}(0)-\overline{I(0)}\right]\left[I_{i}(\tau)-\overline{I(\tau)}\right]}{\sqrt{\sum_{i}\left[I_{i}(0)-\overline{I(0)}\right]^{2}} \sqrt{\sum_{j}\left[I_{j}(\tau)-\overline{I(\tau)}\right]^{2}}}
$$

where indices $i$ and $j$ correspond to CCD pixels and $I_{i}(\tau)$ is the intensity at pixel $i$ as a function of time. $\overline{I(\tau)}$ is the average intensity of the whole CCD image at time $\tau$. The correlation function Eq. 2 equals unity when the image at time $\tau$ is equal to the image at $\tau=0$. It vanishes when the two images are completely uncorrelated. 


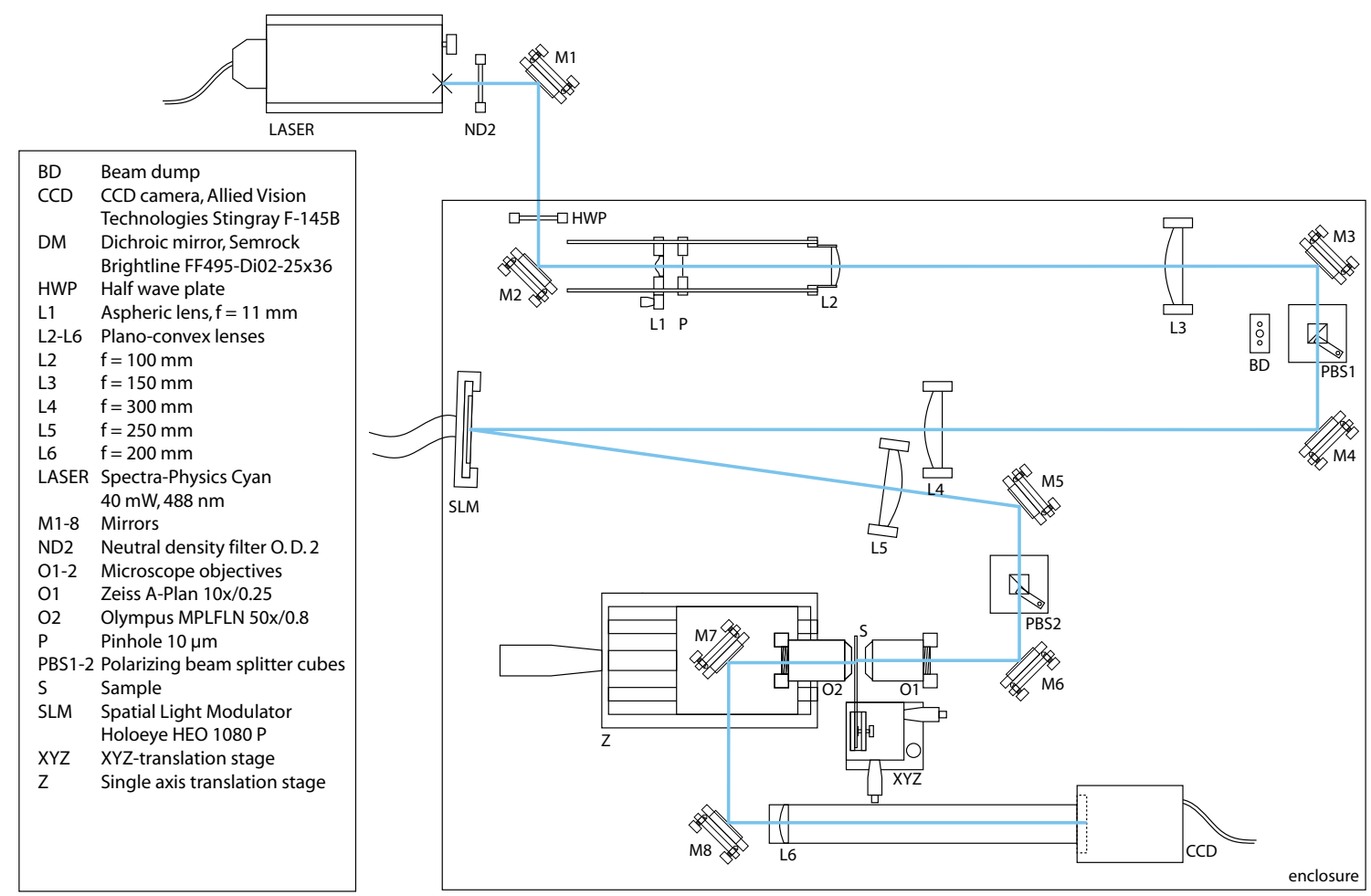

Figure 2. Detailed schematic of the wavefront shaping apparatus.

The speckle decorrelation model that was used to analyze the partitioning algorithm in Ref. 29 assumes that the cross correlation between initial and current speckle patterns decreases exponentially with time. The time associated with this decay is the persistence time $T_{p}$ in Eq. (1). We determine this value experimentally by fitting Eq. (2) with an exponential function. When $T_{p}$ is known, we can use Eq. (1) to find the theoretical maximum enhancement that can be achieved with the algorithm.

\section{EXPERIMENTAL SETUP}

\subsection{Apparatus}

A detailed schematic of the experiment is shown in Fig. 2. A polarized laser beam with a wavelength of $488 \mathrm{~nm}$ is expanded by a factor of 9 using the spatial filter formed by L1, L2 and projected onto the spatial light modulator (SLM) with an additional $2 \times$ magnification. The intensity of the laser is reduced by a neutral density filter and fine adjusted using a combination of a rotatable half wave plate (HWP) and a polarizer (PBS1).

The beam is shaped spatially using a reflective phase-only SLM. The pixels of the SLM are grouped into $50 \times 50$ square segments. The SLM is connected to the digital video interface (DVI) output of a video graphics card in the PC. The lookup table in the SLM hardware was configured so that gray values of 0-255 correspond to phase delays of $0-(255 / 128) \pi$ respectively. The computer sets the phase for each of the segments. The SLM and all other hardware are controlled by custom ActiveX components written in C++. Hardware acceleration (Direct3D) is used to achieve real time (60 frames per second) performance. The components were 'wired together' in the scripting language Python to control different experiments.

A lens and a 10X microscope objective image the surface of the SLM onto the surface of the pupa. The front surface is in the focal plane of microscope objective O1. The back surface of the pupa is imaged onto a CCD camera using objective $\mathrm{O} 2$ and lens L6. We defined a target area on the camera, corresponding to a circle with a diameter of $0.5 \mu \mathrm{m}$ on the sample surface. The computer program integrates the camera intensity over this target area to provide a feedback signal for the algorithm. We choose a shutter time that is equal to the frame interval of the light modulator $(1 / 60 \mathrm{~s})$ to average out phase jitter related to the D/A conversion in the SLM. 


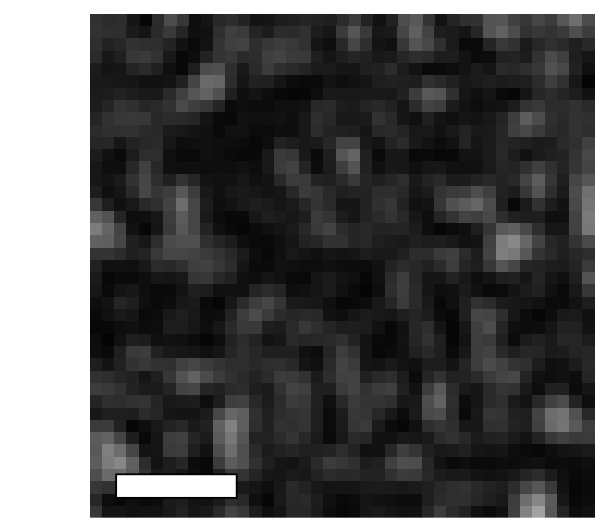

(a)

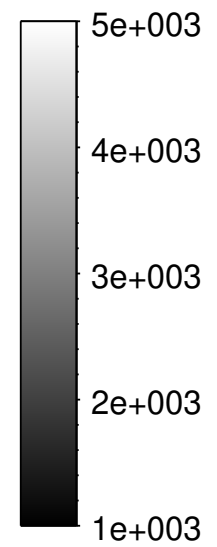

(b)
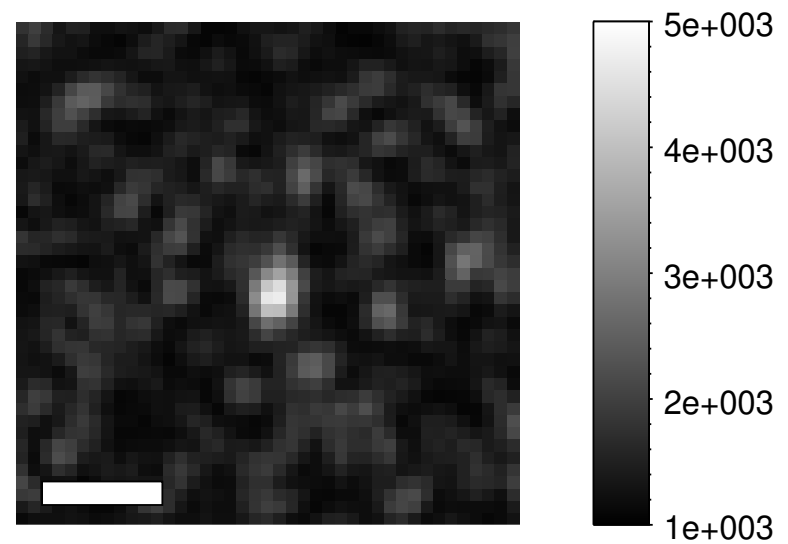

Figure 3. Intensity at the back surface of the pupa. (a) Before optimization only a speckle pattern with a low intensity is visible. (b) After optimization of the incident wavefront, light focuses through the living pupa. Scale bars are $1 \mu \mathrm{m}$ long.

\subsection{Samples}

We chose to use pupae from the Drosophila melanogaster for our experiment. The reason for this choice is twofold. First of all, there is an extremely productive research community that studies the developmental biology of these fruit flies. Using genetic constructs such as fluorescently labeled proteins, much insight in developmental processes has been gathered. Unfortunately, during metamorphosis, where the larva turns into a fly and most of the interesting shape changes take place, the animal is embedded in an opaque pupal case. Although this case can be removed in later stages without killing the animal, in the pre-pupa stage the case is part of the animals skin and cannot be removed without damage. Therefore, there are many potential applications of interferometric focusing for imaging, light-activated gene expression, or opto-thermal manipulation.

Secondly, the Drosophila is a readily available model system for studying biological processes. The flies are easy to handle and breed. Moreover, almost any gene can be knocked out, overexpressed or replaced either systemically or locally when desired. Thanks to the biomolecular toolbox available for Drosophila, it is the ideal system for studying complex multicellular organisms in vivo at the cellular level.

During pupation, the animal is embedded in a pupal case with a thickness of approximately $8 \mu \mathrm{m}$. The casing consists mainly of chitin and proteins ${ }^{34}$ and is highly scattering. The inside of the pupa is less scattering. In the early pupal stage, scattering is dominated by the fat body. During pupation, the heart beat is intermittent and can stop for minutes. ${ }^{35,36}$ This allows us to perform wavefront shaping experiments without the strong movement that is related to the heart beat.

\section{RESULTS}

We placed a pupa of a Drosophila melanogaster in the wavefront shaping apparatus. The pupa was approximately $1 \mathrm{~mm}$ in diameter and $4 \mathrm{~mm}$ long, with an approximately ellipsoid shape. We aligned the objectives so that the SLM was imaged onto one side of the pupa casing. The opposite side of the pupa casing, $1 \mathrm{~mm}$ away from the source, was imaged onto the CCD camera.

When we illuminate the pupa with the spatial light modulator switched off, the CCD camera records a disordered speckle pattern with a weak intensity (see Fig. 3a). The light has passed two layers of strongly scattering cuticle $(8 \mu \mathrm{m}$ each) and the weaker scattering inside of the pupa. Now we run the optimization algorithm, using the intensity from a selected area on the camera as feedback. The algorithm was run continuously for 4000 iterations to make sure that a 'steady state' situation is reached. Figure $3 \mathrm{~b}$ shows the intensity of the transmitted light for the optimized wavefront. Clearly, a sharp focus is formed at the desired location.

The intensity contrast between the focus and the background is a factor three. Considering the fact that we focus light through a living animal with a mainly fluid body, the fact that wavefront shaping is still able to focus light is surprising. It shows that already this prototype wavefront shaping apparatus is fast enough to compensate for some of the movement of 


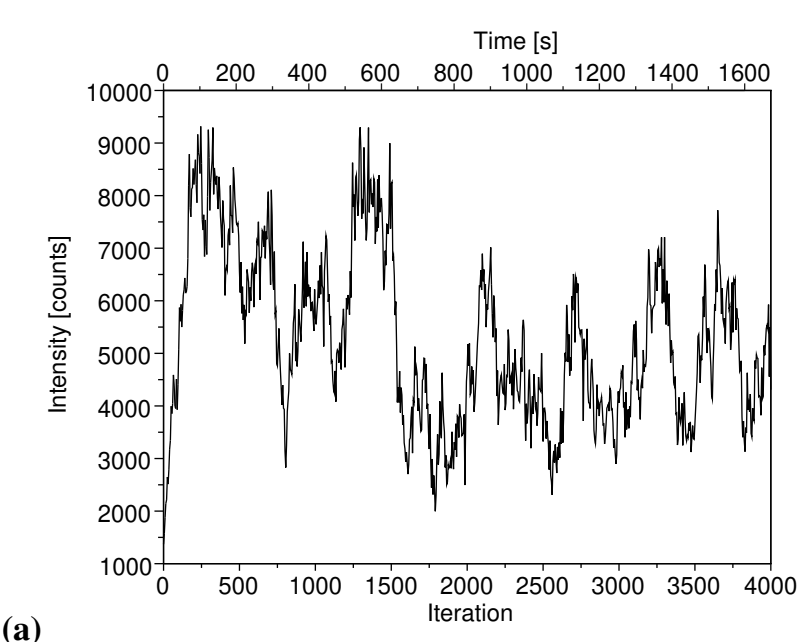

(b)

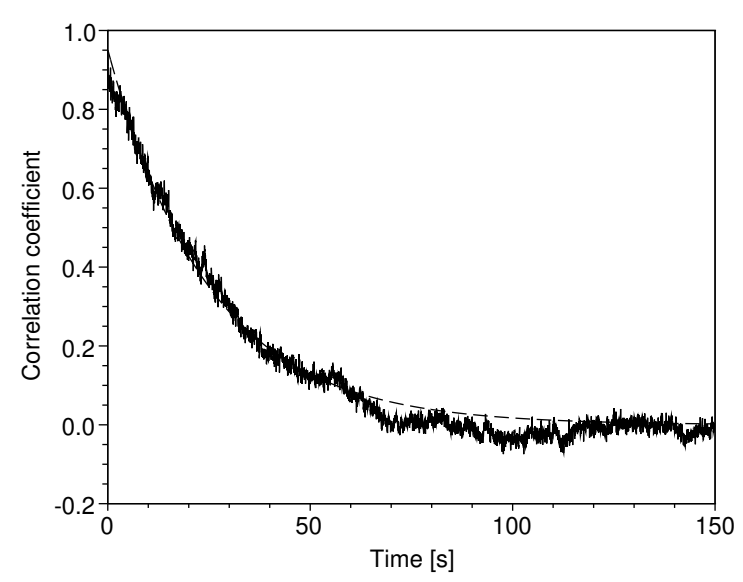

Figure 4. (a) Intensity in the desired focus during the optimization process. After a rapid increase, the intensity fluctuates between 2.2 and 6.8 times the starting intensity. (b) Decorrelation of the speckle pattern. The graph shows the cross correlation coefficient between the current frame and the first frame of the CCD camera. As the speckle pattern changes, the correlation coefficient decreases. The dashed line is a fit with a decay time of 25 seconds.

particles in the animal. Since the expected enhancement scales linearly with the speed of the wavefront shaping apparatus (Eq. (1)), this result stimulates the development of faster setups that will be able to create a focus with an even higher contrast.

We monitored the intensity in the target focus while the algorithm was running. The measurements are shown in Fig. 4a. We observe a rapid, nearly linear increase in intensity from 1370 to 8790 counts during the first 175 iterations. After this rapid 6.4-fold increase in intensity, the intensity in the target focus continues to fluctuate between about 3000 and 9000 counts. The target intensity follows a characteristic pattern. The intensity increases rapidly when the algorithm is optimizing the wavefront. At some point a steady state is reached, where the algorithm is just able to maintain the intensity and continuously adjusts the wavefront in response to moving scatterers. After a while, a 'catastrophe' occurs and the focus is lost partially. Such a catastrophe could coincide with a muscle contraction in the developing pupa or a different event that is isolated in time. Even after a catastrophe, the optimization is not completely lost and the algorithm rapidly rebuilds the focus.

We measured the persistence time of the speckle pattern by switching off the light modulator and recording 26.7 frames per second with the CCD camera. Each frame was correlated with the initial frame using Eq. (2). The results are shown in Fig. 4b. The correlation function is seen to decay nearly exponentially. From an exponential fit, we find a decay time of $T_{p}=25$ seconds. In this time, about 60 iterations of the algorithm can be made, so from Eq. (1) we expect a theoretical enhancement of 30. The fact that we observe only an enhancement of 6.4 can be attributed to the fact that the signal is very noisy, probably because of fast, but weak, fluctuations in the speckle pattern and measurement noise of the camera. Indeed, the algorithm had to trace back its steps 678 out of 4000 times to undo a change that deteriorated the focus.

\section{CONCLUSION AND OUTLOOK}

We demonstrated that wavefront shaping can be used to focus light trough living turbid tissue. A feedback algorithm was able to dynamically adjust the wavefront to compensate for movements inside the specimen. The observed contrast is somewhat lower than the expected maximum value, most likely due to fast fluctuations in the feedback signal. The cause of these fluctuations needs to be investigated further. It might also be possible to find different algorithms that have a lower sensitivity to phase noise. The intensity of the focus is expected to scale linearly with the speed of the wavefront shaping apparatus. Therefore, a faster apparatus will produce a more intense focus and it will extend the applicability of wavefront shaping to more dynamic or thicker systems.

Currently, the limiting factor of the wavefront shaping process is the response time of the light modulator. The current apparatus can cycle the phase from 0 to $2 \pi$ and capture and analyze the CCD images just over two times a second. For- 
tunately, much faster options for light modulation are available. One extremely fast option is acousto-optical modulation. Commercial modulators with modulation frequencies of $10 \mathrm{GHz}$ are available. ${ }^{37}$ Combining multiple modulators in a SLM and tuning their relative phases might prove challenging, but is possible in principle. Already, 24-channel acousto-optical light modulators have been developed for a more 'modest' $100 \mathrm{kHz},{ }^{38}$ which is still a factor of 50000 faster than our setup. Using micromachined membrane mirrors an astounding $10 \mathrm{MHz}$ modulation rate is possible. ${ }^{39}$

Obviously, at such rates the camera needs to be replaced by a fast detector, such as a photodiode and dedicated hardware is needed for calculating the optimum wavefront. Moreover, when the SLM is operated at high speed, sending full frames to the modulator will take considerable bandwidth and processing power. Fortunately, it is possible to use simple algorithms, such as the sequential algorithms discussed in Ref. 29, where in each iteration only a single pixel is addressed. This way, very little calculations or bandwidth is required and control electronics will not form a bottleneck even at speeds of tens of $\mathrm{MHz}$. With a potential speed increase of a factor of $10^{7}$, wavefront shaping can be applied to a variety of specimens, including perfused tissue.

Perhaps even more interesting is the ability to focus light inside turbid tissue. In Ref. 27 it was shown that light can be focused on fluorescent nanoparticles hidden deep inside a strongly scattering medium. This technique will make it possible to use wavefront shaping to focus light on individual living cells tagged with a fluorescent marker. This will pave the way for many exciting applications, for instance capturing cells in vivo using in optical tweezers, locally activating photosensitive chemicals, or even destroying individual cells using thermal effects or phototoxicity.

\section{ACKNOWLEDGMENTS}

We thank Giulia Ghielmetti for experimental support and Ulrike Nienhaus for supplying samples. This work was supported by SystemsX.ch within the framework of the WingX RTD. IMV is supported by a 'Forschungskredit' of the University of Zurich.

\section{REFERENCES}

[1] Cope, M. and Delpy, D. T., "System for long-term measurement of cerebral blood and tissue oxygenation on newborn infants by near infrared transillumination," Med. Biol. Engin. Comput . 26, 289-294 (1988).

[2] Villringer, A., Planck, J., Hock, C., Schleinkofer, L., and Dirnagl, U., "Near infrared spectroscopy (NIRS): a new tool to study hemodynamic changes during activation of brain function in human adults," Neurosci. Lett. 154, 101-104 (1993).

[3] Maki, A., Yamashita, Y., Ito, Y., Watanabe, E., Mayanagi, Y., and Koizumi, H., "Spatial and temporal analysis of human motor activity using noninvasive NIR topography," Med. Phys. 22, 1997-2005 (1995).

[4] Svoboda, K., Denk, W., Kleinfeld, D., and Tank, D. W., "In vivo dendritic calcium dynamics in neocortical pyramidal neurons," Nature 385, 161-165 (1997).

[5] Ntziachristos, V., "Fluorescence molecular imaging," Annu. Rev. Biomed. Eng. 8, 1-33 (2006).

[6] Engelbrecht, C. J., Greger, K., Reynaud, E. G., Kržič, U., Colombelli, J., and Stelzer, E. H., "Three-dimensional laser microsurgery in light-sheet based microscopy (SPIM)," Opt. Expr. 15, 6420-6430 (2007).

[7] Ashkin, A., Dziedzic, J. M., Bjorkholm, J. E., and Chu, S., "Observation of a single-beam gradient force optical trap for dielectric particles," Opt. Lett. 11, 288-290 (1986).

[8] Patrice, T., ed., [Photodynamic Therapy], Comprehensive Series in Photochemical \& Photobiological Sciences, RSCPublishing, London (2003).

[9] Ando, H., Furuta, T., Tsien, R. Y., and Okamoto, H., "Photo-mediated gene activation using caged RNA/DNA in zebrafish embryos," Nature Genetics 28, 317 (2001).

[10] Han, X. and Boyden, E. S., "Multiple-color optical activation, silencing, and desynchronization of neural activity, with single-spike temporal resolution," PLoS One 2, e299 (2007).

[11] Vellekoop, I. M. and Mosk, A. P., "Focusing coherent light through opaque strongly scattering media," Opt. Lett. 32(16), 2309-2311 (2007).

[12] Minsky, M., "Microscopy apparatus." Patent (1961). US3013467.

[13] Chalfie, M., Tu, Y., Euskirchen, G., Ward, W. W., and Prasher, D. C., "Green fluorescent protein as a marker for gene expression," Science 263, 802-805 (1994). 
[14] Huisken, J., Swoger, J., Del Bene, F., Wittbrodt, J., and Stelzer, E. H. K., "Optical sectioning deep inside live embryos by selective plane illumination microscopy," Science 305, 1007-1009 (2004).

[15] Keller, P. J., Schmidt, A. D., Wittbrodt, J., and Stelzer, E. H. K., "Reconstruction of zebrafish early embryonic development by scanned light sheet microscopy," Science 322, 1065-1069 (2008).

[16] Denk, W., Strickler, J. H., and Webb, W. W., "Two-photon laser scanning fluorescence microscopy," Science 248, 73-76 (1990).

[17] Huang, D., Swanson, E. A., Lin, C. P., Schuman, J. S., Stinson, W. G., Chang, W., Hee, M. R., Flotte, T., Gregory, K., and Puliafito, C. A., "Optical coherence tomography," Science 254, 1178-1181 (1991).

[18] Tyson, R. K., [Principles of Adaptive Optics], Academic Press, New York, 2nd ed. (1998).

[19] Yodh, A. and Chance, B., "Spectrocopy and imaging with diffusing light," Phys. Today 48, 34-40 (1995).

[20] Corlu, A., Choe, R., Durduran, T., Rosen, M. A., Schweiger, M., Arridge, S. R., Schnall, M. D., and Yodh, A. G., "Three-dimensional in vivo fluorescence diffuse optical tomography of breast cancer in humans," Opt. Expr. 15, 6696-6716 (2007).

[21] Vinegoni, C., Pitsouli, C., Razansky, D., Perrimon, N., and Ntziachristos, V., "In vivo imaging of drosophila melanogaster pupae with mesoscopic fluorescence tomography," Nature Methods 5, 45-47 (2008).

[22] Fisher, R. A., ed., [Optical phase conjugation], Academic Press, New York (1983).

[23] Yaqoob, Z., Psaltis, D., Feld, M. S., and Yang, C., "Optical phase conjugation for turbidity suppression in biological samples," Nature Photonics 2, 110-115 (2008).

[24] Lerosey, G., de Rosny, J., Tourin, A., and Fink, M., "Focusing beyond the diffraction limit with far-field time reversal," Science 315, 1120-1122 (2007).

[25] Moura, J. M. F. and Jin, Y., "Detection by time reversal: single antenna," IEEE Transactions on Signal Processing 55, 187-201 (2007).

[26] Fink, M., Cassereau, D., Derode, A., Prada, C., Roux, P., Tanter, M., Thomas, J.-L., and Wu, F., "Time-reversed acoustics," Rep. Prog. Phys. 63, 1933-1995 (1999).

[27] Vellekoop, I. M., van Putten, E. G., Lagendijk, A., and Mosk, A. P., "Demixing light paths inside disordered metamaterials," Opt. Expr. 16(1), 67-80 (2008).

[28] Popoff, S. M., Lerosey, G., Carminati, R., Fink, M., Boccara, A., and Gigan, S., "Measuring the transmission matrix in optics : An approach to the study and control of light propagation in disordered media," arXiv: physics.optics/0910.5436v1 (2009).

[29] Vellekoop, I. M. and Mosk, A. P., "Phase control algorithms for focusing light through turbid media," Opt. Comm. 281, 3071-3080 (2008).

[30] Wolf, P. and Maret, G., "Multiple light scattering from disordered media. the effect of Brownian motion of scatterers," Zeitschrift für Physik B 65, 409 (1987).

[31] Pine, D. J., Weitz, D. A., Chaikin, P. M., and Herbolzheimer, E., "Diffusing wave spectroscopy," Phys. Rev. Lett. 60(12), 1134-1137 (1988).

[32] Briers, J. D., "Laser Doppler, speckle and related techniques for blood perfusion mapping and imaging," Physiol. Meas. 22, R35-R66 (2001).

[33] Bissig, H., Romer, S., Cipelletti, L., Trappe, V., and Schurtenberger, P., "Intermittent dynamics and hyper-aging in dense colloidal gels," Phys. Chem. Comm. 6, 21 (2003).

[34] Doctor, J., Fristrom, D., and Fristrom, J. W., "The pupal cuticle of drosophila: Biphasic synthesis of pupal cuticle proteins in vivo and in vitro in response to 20-hydroxyecdysone," J. Cell Biol. 101, 189-200 (1985).

[35] Sláma, K. and Farkaš, R., "Heartbeat patterns during the postembryonic development of drosophila melanogaster," $J$. Insect Physiol. 51, 489-503 (2005).

[36] Sláma, K., "Heartbeat reversal after sectioning the dorsal vessel and removal of the brain of diapausing pupae of manduca sexta (lepidoptera: Sphingidae)," Eur. J. Entomol. 103, 17-26 (2006).

[37] (2009). Thorlabs Catalog item number LN53S.

[38] Yin, S., Leonov, O., Yu, F. T. S., Molotok, V. V., and Kludzin, V. V., "Design and fabrication of a 24-channel acoustooptic spatial light modulator," Appl. Opt. 37, 7482-7489 (1998).

[39] Griffith, G., Haji-saeed, B., Sengupta, S. K., Goodhue, W. D., Khoury, J., Woods, C. L., and Kierstead, J., "Patterned multipixel membrane mirror MEMS optically addressed spatial light modulator with megahertz response," IEEE Phot. Tech. Lett. 19, 173-175 (2007). 